\title{
CULTURAL HERITAGE MONITORING BY LOW-COST GNSS RECEIVERS: A FEASIBILITY STUDY FOR SAN GAUDENZIO'S CUPOLA, NOVARA
}

\author{
R. Barzaghi ${ }^{1}$, M. Reguzzoni ${ }^{1 *}$, C. I. De Gaetani ${ }^{1}$, S. Caldera $^{2}$, L. Rossi ${ }^{3}$ \\ ${ }^{1}$ Politecnico di Milano, Department of Civil and Environmental Engineering (DICA), Milan, Italy \\ (riccardo.barzaghi, mirko.reguzzoni, carloiapige.degaetani)@ polimi.it \\ ${ }^{2}$ Geomatics Research \& Development s.r.l. (GReD), Lomazzo, Italy - stefano.caldera@g-red.eu \\ ${ }^{3}$ Idrogea Servizi s.r.1., Varese, Italy - 1.rossi@idrogea.com
}

Commission II, WG II/8

KEY WORDS: Monitoring, GNSS, Low-cost, Spline Interpolation, Cultural Heritage, San Gaudenzio’s Basilica

\begin{abstract}
:
In this study the cupola of San Gaudenzio's Basilica in Novara, Italy, has been monitored by using two low-cost GNSS receivers located on the East and West side of the spire. Time series of daily solutions for an observation period of about one year have been collected and interpolated by cubic splines. The minimum description length criterion has been used to optimize this interpolation. The results show that the building had an uplift with a maximum amplitude of about $2 \mathrm{~cm}$ during summer. Moreover, from a joint analysis of the two points, one can realize that the uplift is not homogeneous, but the structure made some oscillations (with an amplitude at most of $4 \mathrm{~mm}$ ) when rising up. As for the planimetric coordinates, the two antennas had a slightly different behaviour. The West point showed displacements at most of $1 \mathrm{~cm}$ and solutions with a very high repeatability of the order of few millimeters. The East point had a similar repeatability until a sudden jump occurred, followed by more noisy solutions in all the three directions. This noise degradation slowly dampened, till almost disappearing at the end of the recorded time series. This anomalous behaviour could be attributed to some structural movements. The test was successful in the sense that (1) it was proved that the millimeter accuracy can be reached by using GNSS low-cost receivers installed at San Gaudenzio's cupola, even with a non-perfect sky visibility; (2) such an accuracy is able to show interesting movements of the cupola that can provide information about its stability.
\end{abstract}

\section{INTRODUCTION}

\subsection{GNSS monitoring}

A possible approach for monitoring the risk of structure failures consists in the continuous determination of the coordinates of a set of points so as to describe displacements directly related to deformations and/or subsidences. Nowadays, the use of Global Navigation Satellite Systems (GNSS) for deformation monitoring is a common practice, e.g. for crustal movements, landslides, structure and infrastructure deformations (Kaloop and Li, 2009, Fastellini et al., 2011, Barzaghi et al., 2018). The GNSS monitoring system is typically implemented installing a network of receivers that guarantee a millimeter accuracy, usually computing solutions on daily periods. For the previously mentioned applications, this accuracy is generally required to detect significant movements. Until a few years ago, such a target was achievable just by using geodetic quality GNSS receivers that, due to their cost, limited the application fields and the number of points that could be simultaneously monitored. Nowadays, low-cost GNSS receivers represent a valid alternative to the geodetic ones, providing a similar accuracy with a cost which is about one order of magnitude lower (Cina and Piras, 2014, Biagi et al., 2016, Caldera et al., 2016). Differently from structures like dams or bridges, in case of historical buildings the network design generally has some difficulties. For instance, due to their location and/or limited freedom in choosing where installing GNSS antennas for architectural constraints, sub-optimal sky visibility, frequent multipath phenomena and many other limiting factors can

\footnotetext{
${ }^{*}$ Corresponding author
}

affect the quality of the measurements and must be taken into account in the network design. Of course, each building has its own peculiarities and a generalization is not possible. This work aims at analyzing the feasibility of a low-cost GNSS monitoring systems for cultural heritage preservation, in particular assessing the results of a preliminary study conducted for the cupola of San Gaudenzio's Basilica in Novara, Italy. The idea is to show that the proposed system is able to monitor the monument deformation, thus offering useful data to structural engineering investigations, as well as setting up a low-cost service (Sampietro et al., 2017) to prevent collapse risks through the detection of a precursory change of geometry.

\subsection{San Gaudenzio's cupola}

San Gaudenzio's Basilica is an important monument of Catholic worship in the city of Novara, Italy. The architectural complex consists of three main elements realized in different construction phases: the church, the bell tower and the cupola. The construction dates back to the 16th century, but the most important architectural element of the cathedral is its majestic 121 meter high cupola, designed in 1841 by architect-engineer Alessandro Antonelli. The cupola is one of the symbol of the city and a distinctive sign of its landscape. For its construction, Antonelli decided to use only local materials, to tie it more closely to its place of origin. The structure is entirely in brick, local stone and lime, without the use of iron, making it one of the world tallest masonry buildings. The cupola was finished in 1878, after completion of the spire on its top. The cupola of San Gaudenzio consists of three concentric circular frames contained by an outer casing, completely forcible. In addition, to create an inner bracing, Antonelli used a conical 
shape of internal pillars. He created a cupola with a thin structural shell, only one brick header thick, stiffened by a brick network of meridians and parallels. More in depth, the main skeleton of the structure is made up by a system of ribs, or meridians, stiffened by compression rings along three parallels. Although the external cupola and the internal truncated cone are interconnected, because of the different external forces and loads, the two bodies have their own autonomous static behaviour. However, being both constituted by masonry frames, and therefore transmitting their dead loads by means of a pre-established path, their behaviours are similar. Recent studies on the geometry and the mechanics of the cupola (Corradi et al., 2009) revealed that the system of principal and secondary ribs, which connects the two stiffening rings, allows it to accommodate small variations of the initial state of equilibrium without the risk of causing a kinematic collapse. In the years following the completion of the church, it began to show signs of structural failure, already noticed during the early stages of construction. The foundations were recognized as too shallow and the pillars did not have a sufficient base to unloading the weight of the cupola. For this reason, the architect made a hollow sub-foundation using masonry frames, expanding the base of the structure using inverted arches, and thereby creating a kind of pillared gallery. Antonelli's foundations pushed under the old foundations up to the hard layer of solid clay, five meters below the floor of the basilica. In addition, new foundations interested not only the four pillars, but also the eight wings of ramming. The work ended in 1887 but subsequently, concrete reinforcement to the spire and other work at Antonelli's arches were carried out by engineer Arturo Danusso in the 30's of the 20th century. In order to weld the iron belts, in the absence of welding torch, sulphur was set fire. This, however, in the most hidden parts did not receive enough oxygen and was not completely burnt. Then, already in the early 20th century the remaining sulphur gave rise to a sulphuric solution due to precipitation. This, penetrating the granite, weakened the adhesion of the granules that make it up. This disintegration continued uninterruptedly, causing the fall of fragments of granite on the roofs of the church. The sulphur action resulted more harmful in correspondence to the edges of the bases, on the capitals and generally on the more elaborated and protruded parts. Danusso did not realize the risk brought by welds made with sulphur. In fact, he thought that the breaking of the capitals of the spire was due to instability of the structure, insufficiently rigid, therefore attributing to the wind forces the deformation of the spire and the inevitable fall of the pieces on the roof of the church. Danusso did not understand that the cause was not physics but chemistry. On this misunderstanding, he based the intervention of consolidation and strengthening of San Gaudenzio's spire on reinforced concrete. The work started in 1931, by acting on the upper part of the spire. Danusso decided to maintain the exterior unchanged and built a concrete core cable internally to give rigidity to an externally unchanged structure. At this point, the first stylobate and peristyle were weighted by the oscillations of the monolith above. Clearly, the regime of new oscillations was very different from the first one, since, if before each gust of wind caused small deflections on each node, now all the stresses flowed into the base of the second stylobate, increasing moments at that point. So it was that between 1932 and 1934, after the first intervention, the fractures of the inner capitals of the first stylobate of the spire, dividing them into two parts, were determined. The work of consolidation of the cupola continued in 1937. The concrete core cable throughout the lower part of the spire continued, including three little cupolas inside the original one. Voids behind pendentive were filled and the existing pillars were improved, because of concrete reinforcements had to work at double of the load. At the end of the intervention, the weight of the spire, above the cupola, was almost doubled. If before, at the wind action, Antonelli's spire reacted with a deformation work, now the rigid spire transmitted the increased weight to the elastic lower structure, thus amplifying the effect of the wind blow. Practically, once the interventions were completed, the structure gravitational center rose and a greater instability in the structure was created. Then San Gaudenzio's Basilica was closed for almost a decade due to a possible structural failure of the four pairs of arches, that were carrying the cupola. The last consolidation operations took place in 1946. In correspondence to the four pendentives, the high relief of the evangelists were taken away and a concrete cast was made. Furthermore, in correspondence of the ring of the rib sets of the first inner cupola a reinforced concrete ring was built, hiding the existing decorations. Eventually, as far as the four pairs of Antonellian steel rods are concerned, new rods with a bigger diameter were added. In recent years, after the damaging interventions of 1931 and 1937, several doubts regarding San Gaudenzio's spire stability rose (Calderini and Pagnini, 2014). In fact, even though structural failure not yet occurred, after these invasive consolidations the structure became rigid and fragile. Therefore, it was asked to study spire oscillations along with the definition of its verticality, thus justifying the installation of modern monitoring systems, like the GNSS one, to early detect possible small structural failures, cracks or oscillations.

\section{EXPERIMENTAL TEST}

\subsection{Installation}

As for the feasibility test under study, two GPS antennas were located on the platform above the second peristyle of San Gaudenzio's spire (see Figure 1). One of them was located toward West, while the other one towards East. Both antennas had the sky visibility partly occluded by the spire body. The LEA-6T u-blox receivers included in the evaluation kit EVK-6T were used for the test. They are GPS L1-only receivers providing raw code and phase data in binary format, through a COM port. The free and open-source goGPS software (Realini and Reguzzoni, 2013, Herrera et al., 2016) running on a notebook was used to record the data stream, which was then transmitted to a server via GSM connection. The patch antenna ANN-MS-0-005 included in the development kit was replaced by more efficient antennas, namely a Tallysman TW3740 for the West point and a Tallysman TW3742 for the East one. The latter offers a stronger protection against noise thanks to an additional filtering. With the aim of respecting Antonelli's architecture, made of white granite, the antennas were fixed to the concrete basement through two stainless steel supports, as shown in in Figure 2. For the realization of the antenna support, stainless steel AISI 304 X5CrNi 18-10 was used in order to minimize thermal noise thanks to its very low thermal expansion coefficient of $0.0165 \mathrm{~mm} / \mathrm{m}^{\circ} \mathrm{C}$. Considering a possibile variation of temperature of $50^{\circ} \mathrm{C}$ during the year and the length of the supports of $65 \mathrm{~cm}$, the maximum elongation would be of $0.536 \mathrm{~mm}$, one order of magnitude smaller than the expected oscillations of the spire. 


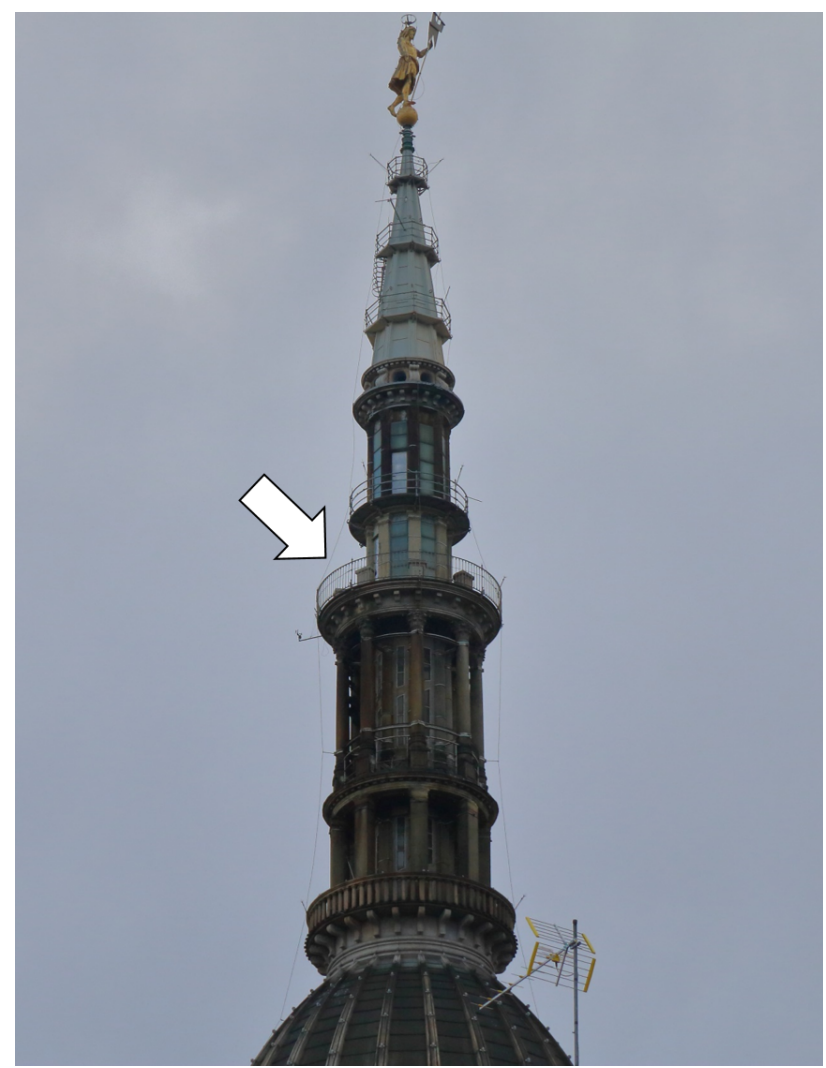

Figure 1. San Gaudenzio's spire; the arrow indicates the second peristyle where the antennas are located

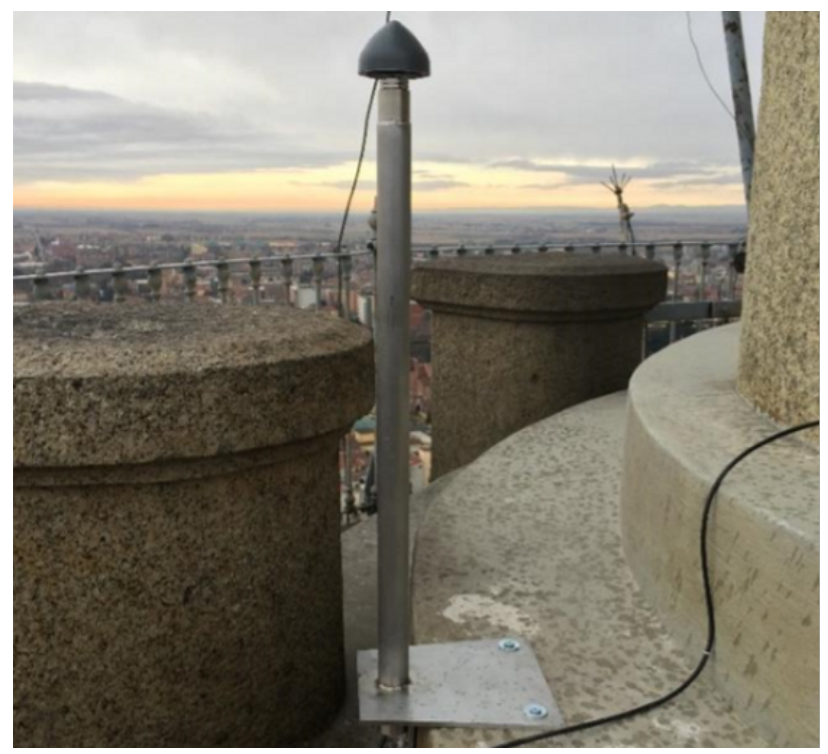

Figure 2. Monumentation of one of the two antennas on the concrete basement

\subsection{GPS processing}

The processing was performed by the Bernese software (Dach et al., 2015), considering short baselines with respect to the Novara GNSS permanent station of the SPIN network (Servizio di Posizionamento INterregionale), located at less than $1 \mathrm{~km}$ from San Gaudenzio's Basilica. The time series of the East-North-Up coordinates for the two monitored points refer to daily solutions for an observation period from 4th January 2018 (epoch 3) to 27th February 2019 (epoch 422), with some data gaps due to data acquisition and transmission problems. The time series of each point refer to the point coordinates of the first day, thus displaying variations with respect to these initial positions. The Bernese processing was carried out with a sampling rate of 30 seconds, corresponding to the data rate of the Novara permanent station, and with a cut-off angle of 10 degrees. Ultra-rapid GNSS ephemerides were used in order to simulate a near-real time processing. No tropospheric parameters were estimated due to the short baseline length. The phase ambiguities were determined by the SIGMA algorithm. Considering the partial sky visibility of the two points on the spire, a multipath-mitigating preprocessing was carried out on the raw GPS data before the Bernese processing. This was performed by proprietary algorithms of goGPS software of GReD s.r.l. company. Figures 3 and 4 show the raw GPS data Signal-to-Noise Ratio (SNR) of the two points for the epoch 137.

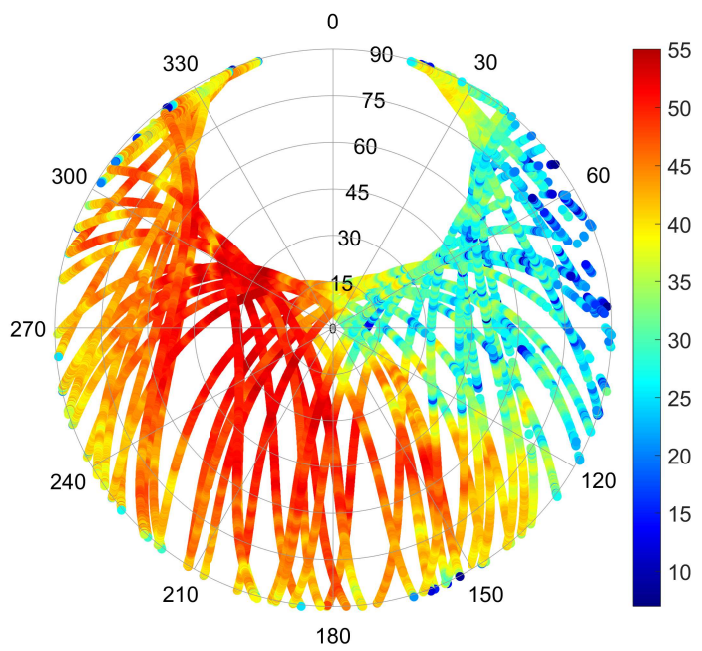

Figure 3. Sky plot of the SNR of the data collected by the West antenna on epoch 137

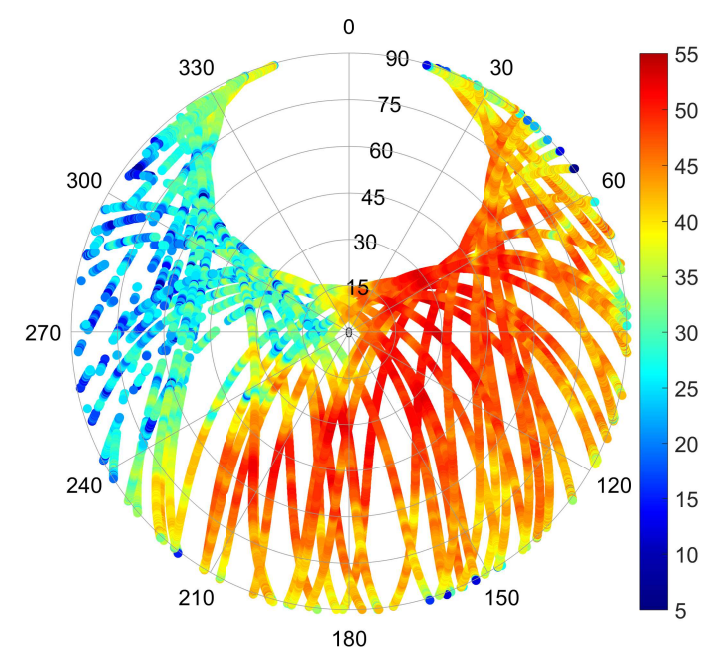

Figure 4. Sky plot of the SNR of the data collected by the East antenna on epoch 137 


\subsection{Time series processing}

The time series of the daily solutions of the two antennas have been processed in order to filter out the main trends of the displacements. These trends have been modelled by interpolating the daily solutions with cubic splines (Brovelli et al., 2001), separately for each component East, North, Up. The splines used for fitting the time series are equally spaced in time and their number have been determined by means of the Minimum Description Length (MDL) criterion. It is based on the principle that the number of parameters defining the interpolating function can be compressed by exploiting the signal regularity in a given dataset (Grunwald, 2007). The MDL criterion evaluates the trade-off between the model prediction residuals and the complexity of the parametric interpolating function. Under the hypothesis of observations with Gaussian errors and known variance, the MDL criterion leads to choose the interpolating function minimizing the quantity:

$$
\mathrm{MDL}=\frac{\ln (N) q}{N}+\ln (\mathrm{SSR})
$$

where $\quad N=$ number of observed data

$q=$ number of model parameters

SSR $=$ Sum of Squared Residuals.

With such an approach, the minimum number of nodes where locating the cubic splines between the beginning and the end of the considered period has been determined, obtaining the long wavelength signal describing the smooth displacements recorded by the antennas. Regarding the West antenna, Figure 5 shows the normalized MDL indexes computed on the basis of 365 observed values and a number of nodes varying between 2 and 15 for the East, North and Up directions, respectively. The normalization has been computed dividing by the maximum value. The minimum MDL value has been obtained interpolating the East displacements with 10 splines, the North displacements with 13 splines and the Up displacements with 8 splines. Considering that the time span between the first and the last observed values was 420 days long, interpolating the time series with such nodes corresponds to modelling the signal with a temporal resolution of 42 days in the East direction, about 32 days in the North direction and about 52 days in the Up direction. Figures 6, 7 and 8 show the observed time series and the corresponding interpolating models.

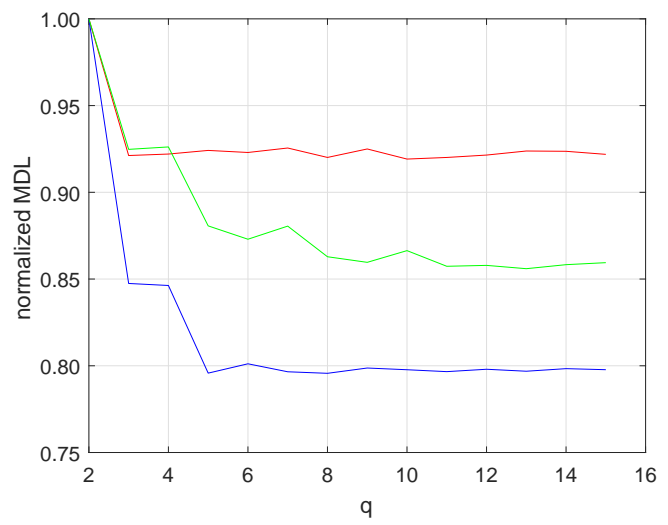

Figure 5. MDL index computed for the West antenna time series in the East (red line), North (green line) and Up (blue line) components

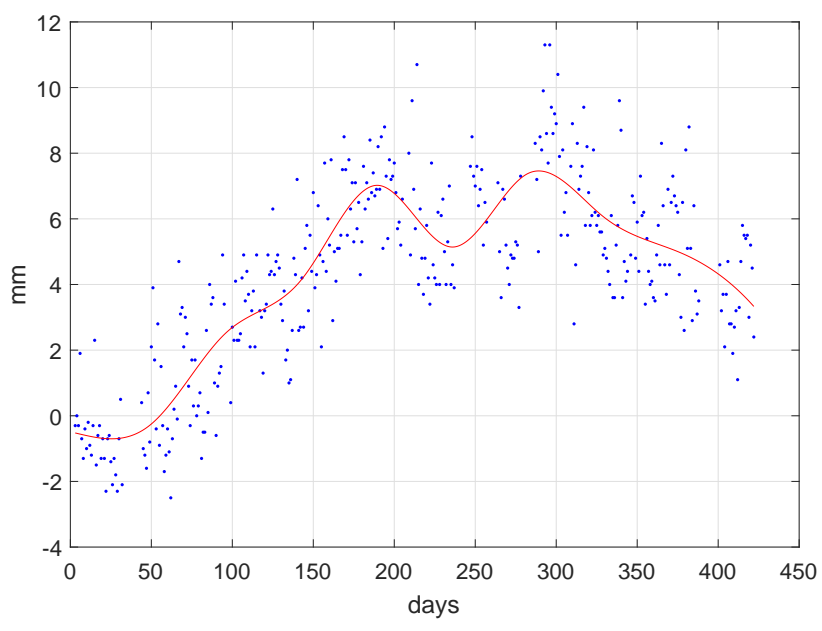

Figure 6. West point time series, East component. Observed values (blue dots) and model (red line)

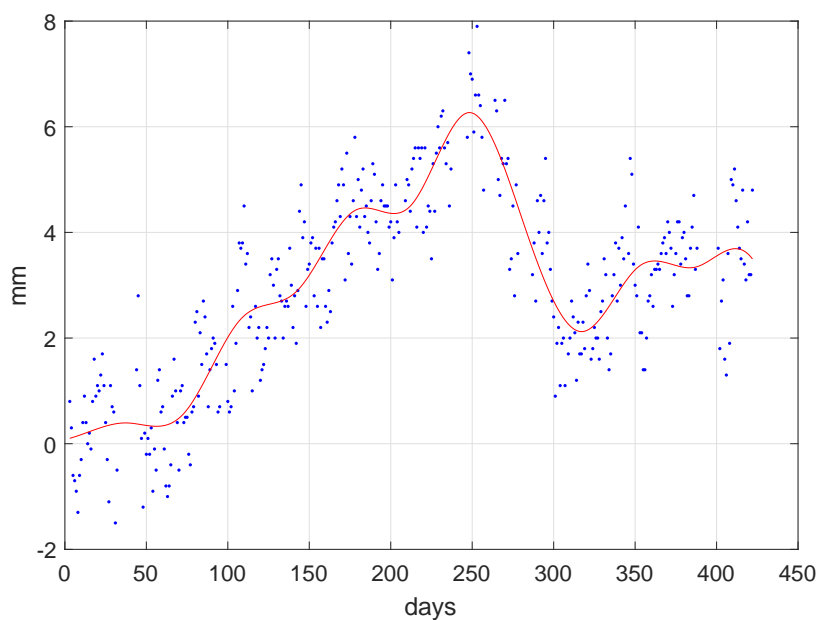

Figure 7. West point time series, North component. Observed values (blue dots) and model (red line)

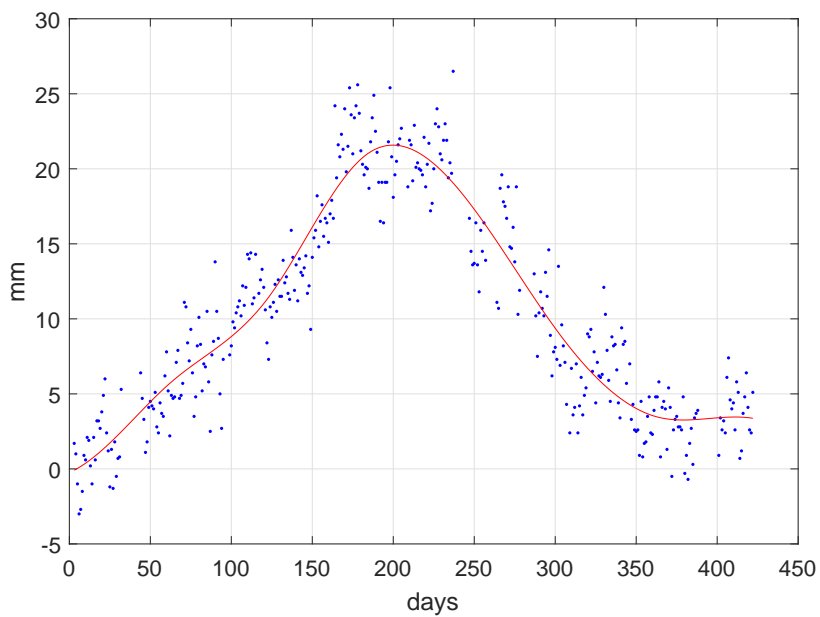

Figure 8. West point time series, Up component. Observed values (blue dots) and model (red line) 


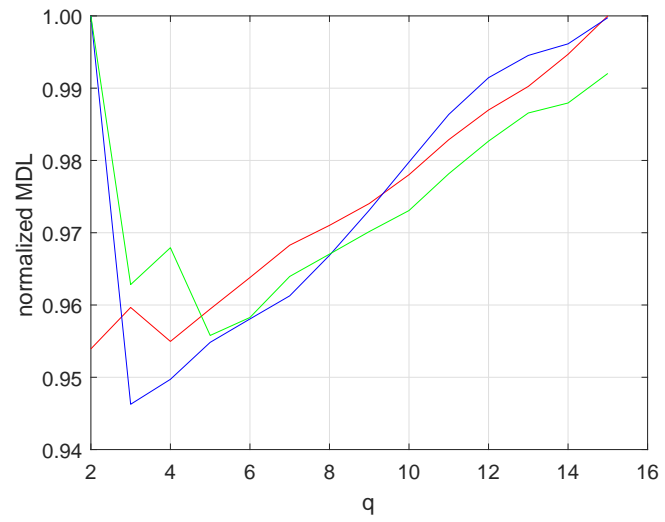

Figure 9. MDL index computed for the East antenna time series in the pre-discontinuity period. East (red line), North (green line) and Up (blue line) components

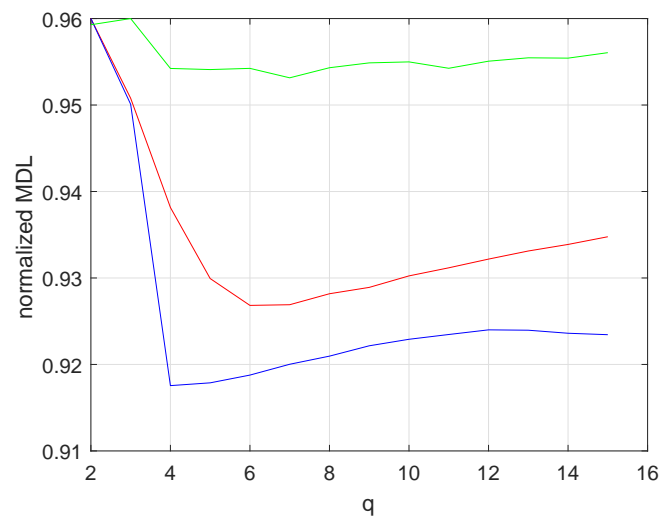

Figure 10. MDL index computed for the East antenna time series in the post-discontinuity period. East (red line), North (green line) and Up (blue line) components

The processing of the East antenna time series required a particular treatment due to a sudden "jump" clearly recorded in the East direction on 4th May 2018 (observed epoch 123). Due to this discontinuity, the three East, North and Up time series have been split into two sub-periods (120 and 300 days long, respectively) and separately considered. Figures 9 and 10 show the normalized MDL indexes computed for the East, North and Up directions on the basis of 106 observed values in the period before the discontinuity, 252 observed values in the subsequent period and a number of nodes varying between 2 and 15. According to the definition of the MDL index (see Eq. 1), with a smaller number of observed values $N$ the penalty introduced by increasing the number of parameters $q$ is more significant, making easier the detection of the minimum MDL value. In the East direction the best interpolation resulted with 4 and 6 nodes for the first and second periods, corresponding to periodicities of 30 and 50 days, respectively. In the North direction it resulted with 5 and 7 nodes ( 24 and about 43 days), while in the Up direction with 3 and 4 nodes (40 and 75 days). Figures 11, 12 and 13 show the observed time series and the corresponding interpolated models for the East antenna.

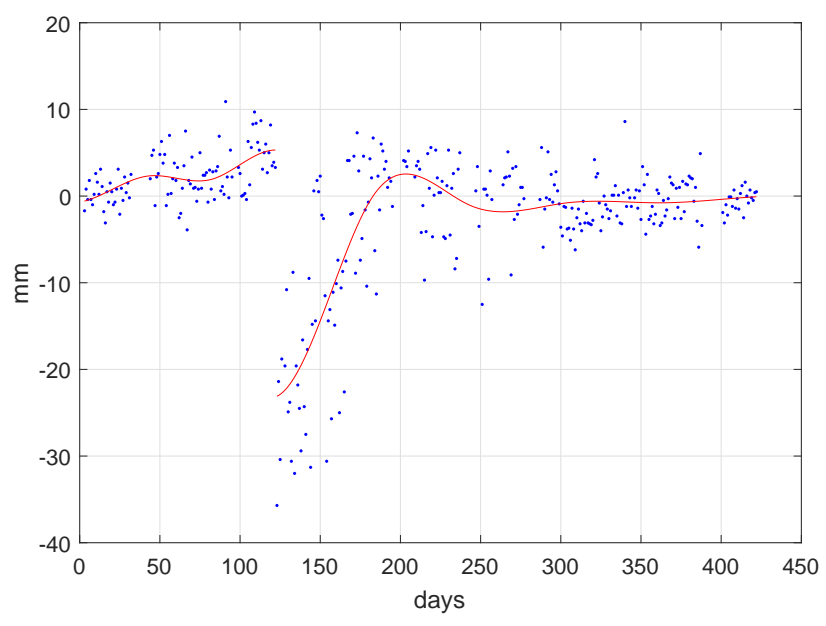

Figure 11. East point time series, East component. Observed values (blue dots) and model (red line)

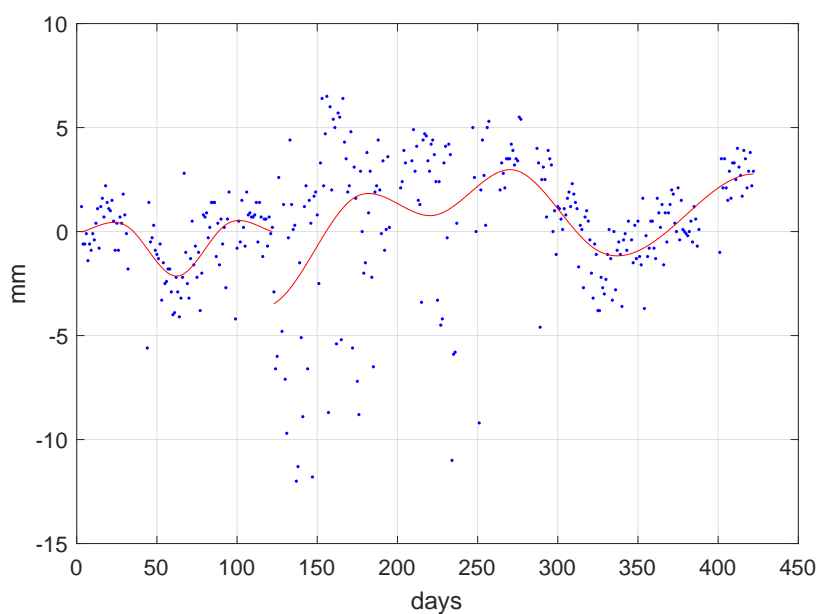

Figure 12. East point time series, North component. Observed values (blue dots) and model (red line)

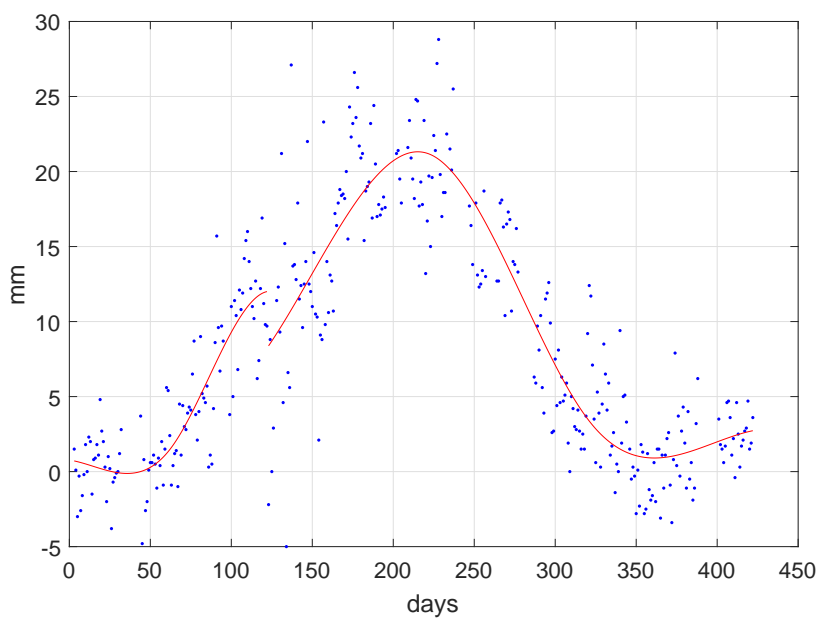

Figure 13. East point time series, Up component. Observed values (blue dots) and model (red line) 


\section{RESULTS}

The results show that the building had an uplift with a maximum amplitude of about $2 \mathrm{~cm}$ occuring during summer. This uplift is clearly correlated with the environmental temperature. In fact the linear correlation coefficient between the daily average temperature (measured by a meteorological station managed by the regional agency for environmental protection and placed few hundreds of meters from the antennas) and the Up component time series is 0.88 and 0.75 for the West point and the East point, respectively. Furthermore, there is no significant residual uplift after one year of observations. Moreover, from a joint analysis of the two points, one can realize that the uplift is not homogeneous, since the structure made some oscillations (see Figures 8 and 13), in particular during the first 50 epochs, with an amplitude at most of 4 $\mathrm{mm}$. Note that the modelled discontinuity in the Up direction of the East antenna results of the same amplitude, leading to consider it plausible. As for the planimetric coordinates, the two antennas have a slightly different behaviour. The West point shows total displacements of at most $1 \mathrm{~cm}$ and solutions with a very high repeatability of the order of 1-2 $\mathrm{mm}$ (see Figures 6 and 7). The East point has a similar repeatability till epoch 123 , then there is a jump of about 2-3 $\mathrm{cm}$ in the East coordinate, followed by more noisy solutions in all three components (see Figures 11, 12 and 13). This noise degradation slowly damps, almost disappearing at the end of October 2018 (epoch 300). This anomalous behaviour can be attributed to some structural movements or to some receiver/antenna problems. Note that in the period around the jump epoch, the East and North coordinates of the West antenna are systematically increasing. Figures 14 and 15 show the $2 \mathrm{D}$ displacements of the two antennas. The West antenna shows a predominant shift along the North-East direction by about $8 \mathrm{~mm}$ in the first half of the observation period. In the second half it seems to randomly move, however tending to the initial position. At the end of the observation period a residual displacement of $4 \mathrm{~mm}$ remains between the initial and the final positions. Regarding the East antenna, the movement is prevailingly in the North-South direction (oscillations of about $3 \mathrm{~mm}$ of amplitude) at both the beginning and the end of the observation period. In the middle, the movement is dominated by the sudden shift of $2.8 \mathrm{~cm}$ mainly in the West direction and the consequent quick return close to the initial position.

A joint analysis has been conducted on the relative distance between the two antennas, as well as the azimut and zenit change of the line joining them. The azimut is the clockwise orientation angle with respect to the North direction, while the zenit is the anticlockwise angle with respect the horizontal plane. Regarding the relative distance, displayed in Figure 16, it oscillates (peak to peak) with amplitude of about $2.5 \mathrm{~mm}$ before the East point sudden displacement. This displacement causes the two points to be closer by about $2.8 \mathrm{~cm}$. However, during the subsequent observation period, the relative distance slowly increases tending to the initial one. Before the end of the observed period, $5 \mathrm{~mm}$ of residual deformation have not been compensated, yet. This residual deformation can be mainly ascribed to the West antenna (see Figure 14). Considering the azimut (see Figure 17), oscillations of about 0.4 degrees (peak to peak) are displayed and the discontinuity causes a change of 0.8 degrees in the clockwise direction. However, the time series still confirms a slow tendency to the initial state. Also the zenit plot (Figure 18) shows small oscillations of about 0.2-0.3 degrees, with a similar behaviour in the periods before and after the discontinuity.

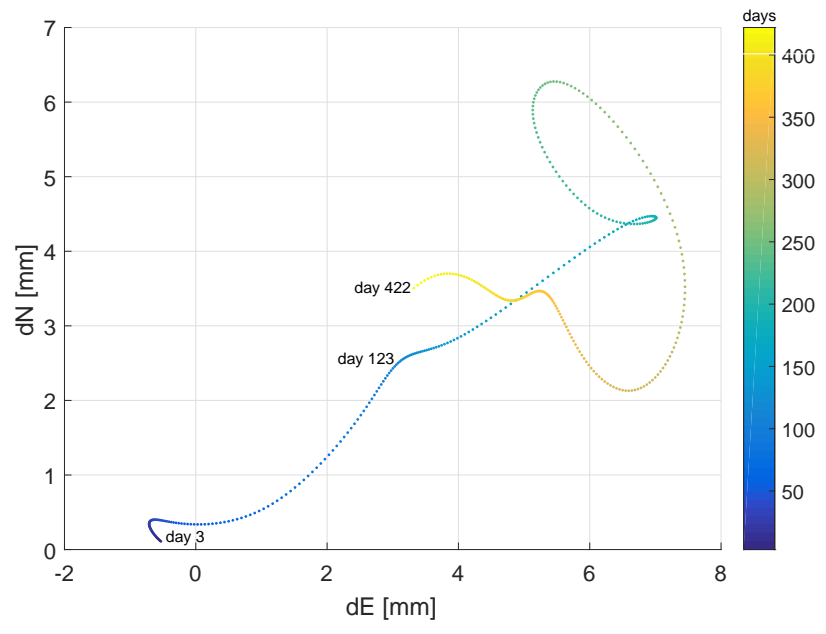

Figure 14. East-North 2D scatter of the modelled West point movements in the observed period

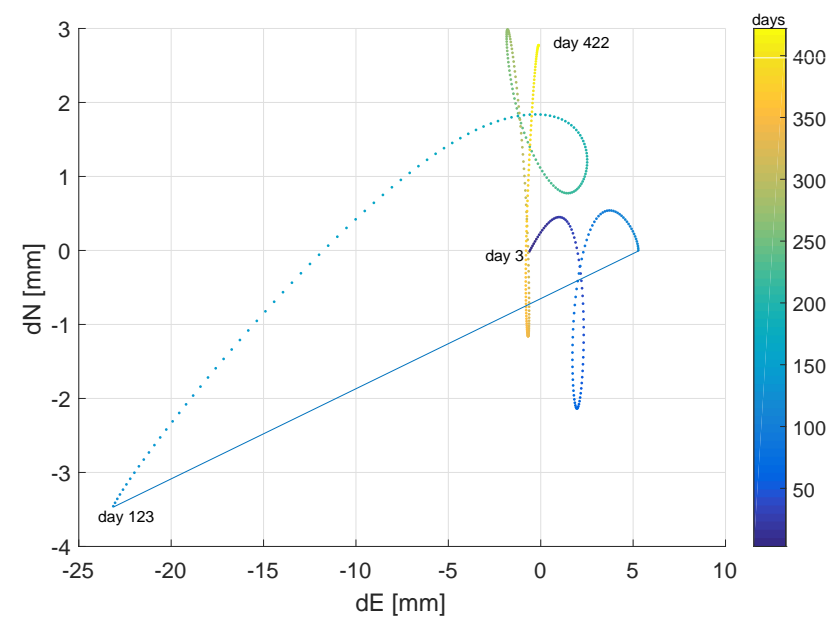

Figure 15. East-North 2D scatter of the modelled East point movements in the observed period

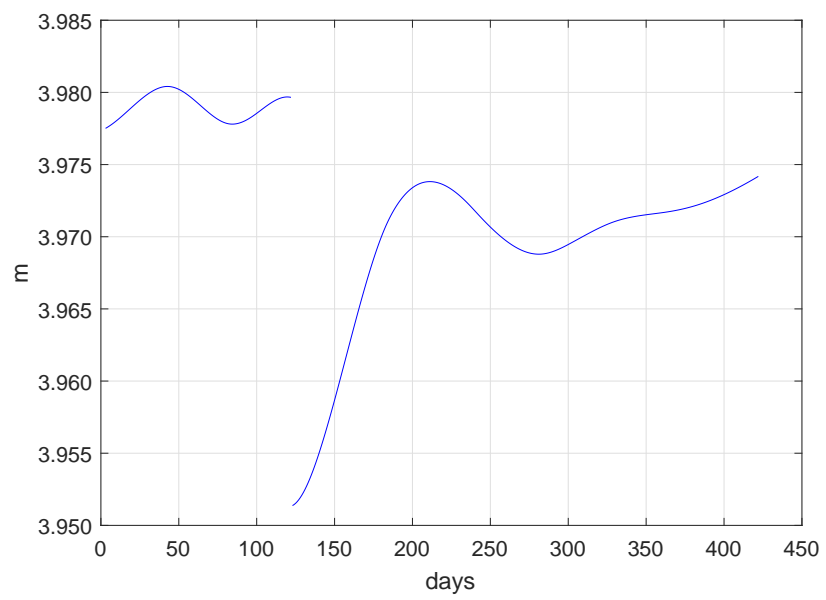

Figure 16. Modelled relative distance between the West and East points during the observed period 


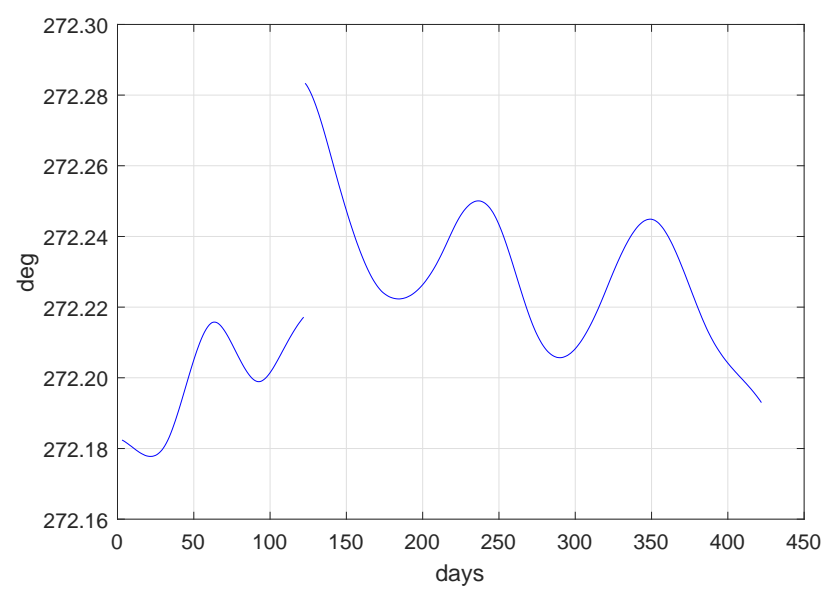

Figure 17. Modelled azimut of the line connecting the

West and East points during the observed period

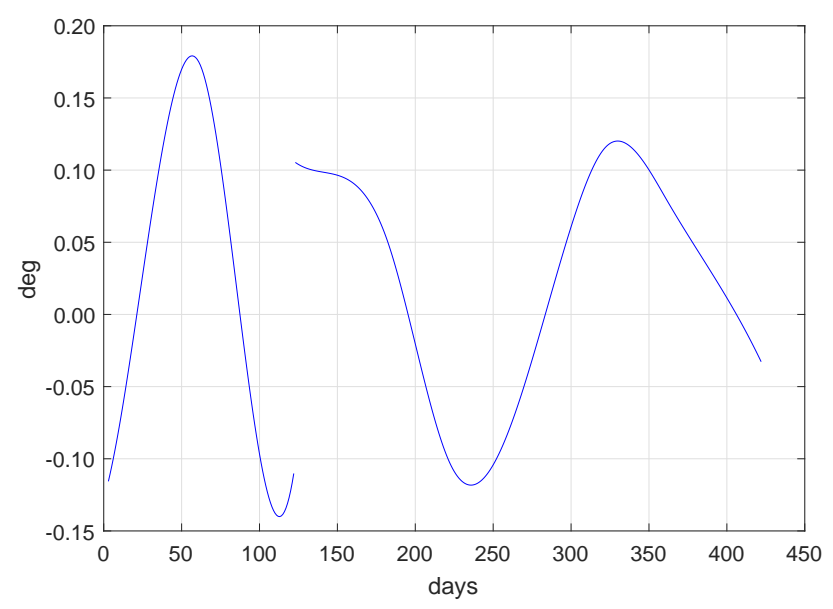

Figure 18. Modelled zenit of the line connecting the West and East points during the observed period

It is interesting to analyze the residuals of the observations with respect to the computed models, in particular their local variability. Figures 19, 20 and 21 show the root mean square (RMS) of the residuals computed on a moving window of 21 days for the East, North and Up components, respectively. The plots clearly highlight that the acquisitions on the East point are generally more noisy than the ones on the West point and that there is a significant deterioration of the solution repeatability as soon as the discontinuity occurs on the East point. Such a deterioration has a peak just after epoch 123 and rapidly reduces around epoch 200. After that, another peak is registered, slowly reducing by the end of the observation period. This could be an indication of a further discontinuity with a small amplitude and therefore less visible when looking at the time series. The detection of the epoch and the amplitude of this possible discontinuity requires further investigations that could exploit more sophisticated techniques (Benciolini et al., 2012). Note that the same general behaviour can be seen in all the three components but with different relative amplitudes. Considering as reference the mean RMS of the residuals before epoch 123, it is noticed that in all the three components the first peak is about 3.5 times bigger, while the second one is still 3.5 times bigger in the North component but just 1.5 times bigger in the East and Up components.

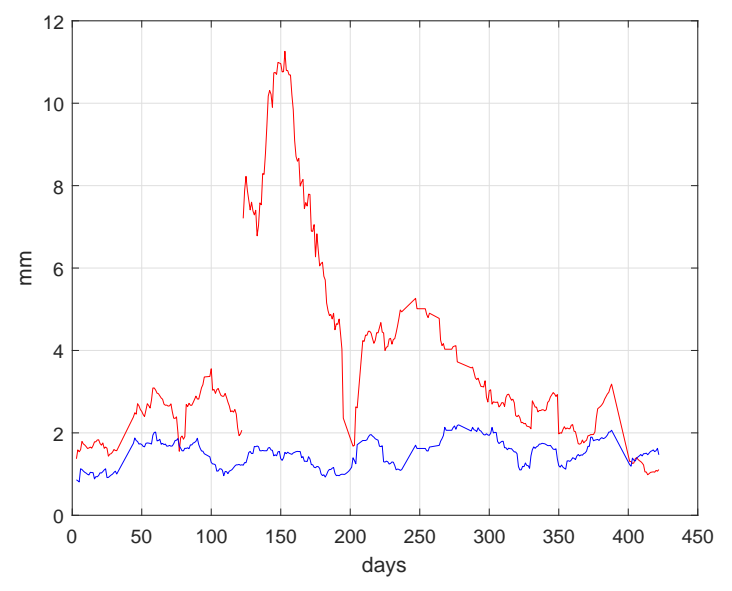

Figure 19. Moving RMS of the residuals of the modelled positions in the East component for the East point (red line) and the West point (blue line)

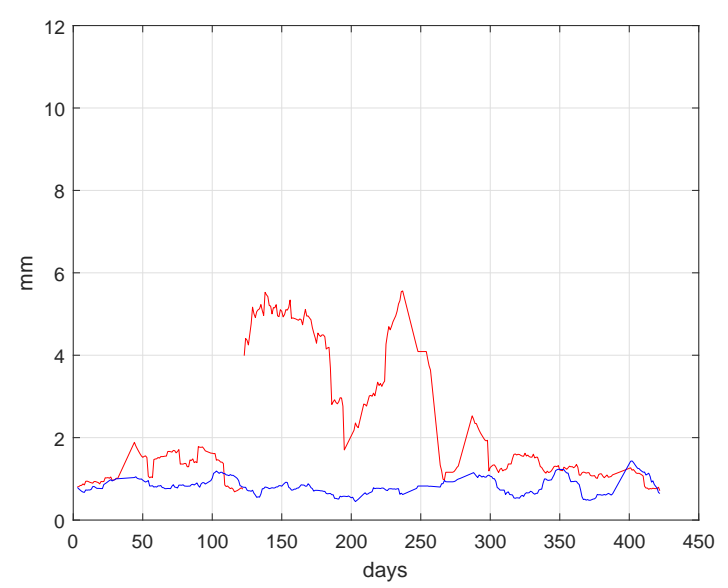

Figure 20. Moving RMS of the residuals of the modelled positions in the North component for the East point (red line) and the West point (blue line)

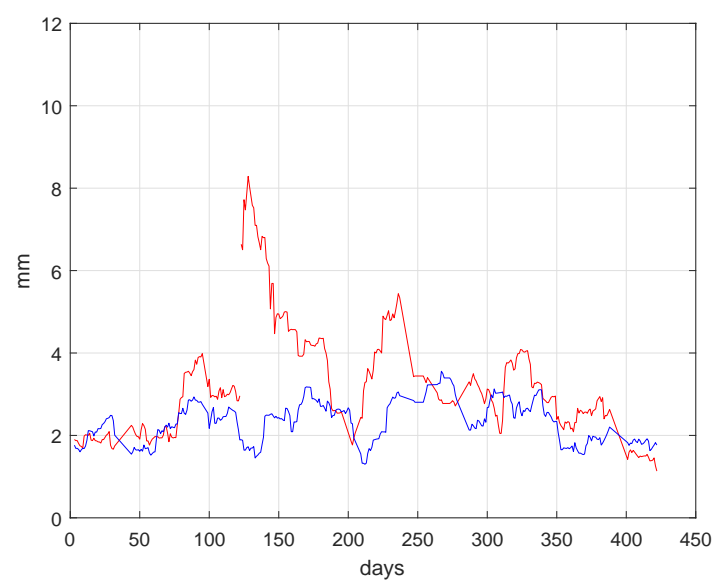

Figure 21. Moving RMS of the residuals of the modelled positions in the Up component for the East point (red line) and the West point (blue line) 
Finally, Figure 22 shows the three dimensional formal errors (again averaged on a moving window of 21 days) associated to the computed daily GPS solution by the Bernese software. As one can see, the East receiver has a formal error about 1.5 times bigger than the West receiver and this could be due to the different sky visibility or quality of the antenna. However, the formal error is quite stable for both of them and for the entire observed period, leading to exclude that the found anomalies was caused by instrument malfunctions and inducing to ascribe them to structural movements and/or deformations.

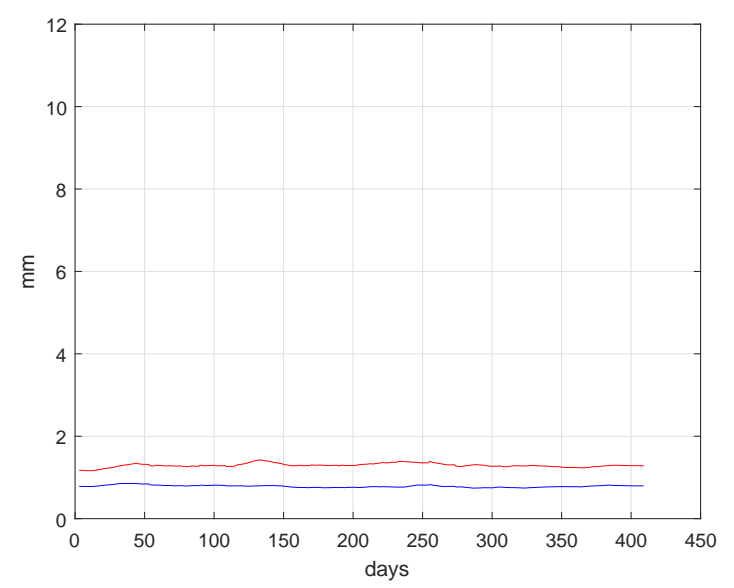

Figure 22. 3D formal error standard deviations for the East point (red line) and the West point (blue line)

\section{CONCLUSIONS}

In this work, low-cost GNSS receivers have been used to monitor the cupola of San Gaudenzio's Basilica in Novara, Italy. This preliminary study has been based on the analysis of the time series of daily solutions of two points, located on the East and West side of the spire. The analyzed time series cover an observation period of about one year. Smooth displacements have been modelled by interpolating the observations with cubic splines, whose spacing has been determined according to the Minimum Description Length criterion. On the basis of this interpolation, it can be concluded that the coordinate time series collected by GNSS low-cost receivers have generally a millimeter accuracy, even with a non-perfect sky visibility. As a consequence, movements, oscillations and deformations of the spire of San Gaudenzio's cupola have been clearly identified. The main motion is obviously in the vertical direction (about $2 \mathrm{~cm}$ ) due to environmental temperature changes. In the horizontal direction, movements are much smaller and of the order of some millimeters, apart from a centimeter discontinuity that occured in May 2018. Generally, the two monitored points do not move fully coherently one another. Oscillations of few tenths of degrees are detected for both the azimut and the zenit angles. The structure also shows some deformations, since the distance between the two points changes in time, with a maximum variation of about $2.8 \mathrm{~cm}$ after the discontinuity and a residual deformation of $5 \mathrm{~mm}$ at the end of this preliminary study period. Summarizing, the test is judged to be successful, confirming the potentiality of a low-cost GNSS monitoring system also in the case of historical buildings for cultural heritage preservation. A more extensive analysis, in terms of monitoring period as well as completeness and reliability of the monitoring system, is recommended for the cupola of San Gaudenzio's Basilica in the future.

\section{REFERENCES}

Barzaghi, R., Cazzaniga, N., De Gaetani, C., Pinto, L. and Tornatore, V., 2018. Estimating and comparing dam deformation using classical and gnss techniques. Sensors 18(3), pp. 756.

Benciolini, B., Reguzzoni, M., Venuti, G. and Vitti, A., 2012. Bayesian and variational methods for discontinuity detection: theory overview and performance comparison. International Association of Geodesy Symposia 137, pp. 147-152.

Biagi, L., Grec, F. and Negretti, M., 2016. Low-cost gnss receivers for local monitoring: experimental simulation, and analysis of displacements. Sensors 16(12), pp. 2140.

Brovelli, M., Reguzzoni, M., Sansó, F. and Venuti, G., 2001. Modelli matematici del terreno per mezzo di interpolatori a spline. Bollettino SIFET special issue 2/2001, pp. 55-80.

Caldera, S., Realini, E., Barzaghi, R., Reguzzoni, M. and Sansó, F., 2016. Experimental study on low-cost satellite-based geodetic monitoring over short baselines. Journal of Surveying Engineering 142(3), pp. 04015016.

Calderini, C. and Pagnini, L., 2014. The debate on the strengthening of san gaudenzio dome in novara (1931-1945): a contribution to the history of wind engineering. Proceedings of the 13th Conference of the Italian Association for Wind Engineering IN-VENTO 2014.

Cina, A. and Piras, M., 2014. Performance of low-cost gnss receiver for landslides monitoring: test and results. Geomatics, Natural Hazards and Risk 6(57), pp. 3-20.

Corradi, M., Filemio, V. and Trenetti, M., 2009. Antonelli's dome for san gaudenzio: Geometry and statics. Nexus Network Journal 11(2), pp. 243-256.

Dach, R., Lutz, S., Walser, P. and Fridez, P., 2015. Bernese GNSS Software, Version 5.2. Astronomical Institute, University of Bern, Bern, Switzerland.

Fastellini, G., Radicioni, F. and Stoppini, A., 2011. The assisi landslide monitoring: a multi-year activity based on geomatic techniques. Applied Geomatics 3(2), pp. 91-100.

Grunwald, P., 2007. The Minimum Description Length Principle. MIT Press.

Herrera, A., Suhandri, H., Realini, E., Reguzzoni, M. and de Lacy, M., 2016. gogps: Open-source matlab software. GPS Solutions 20(3), pp. 595-603.

Kaloop, M. and Li, H., 2009. Monitoring of bridge deformation using gps technique. KSCE Journal of Civil Engineering 13(6), pp. 423-431.

Realini, E. and Reguzzoni, M., 2013. gogps: open source software for enhancing the accuracy of low-cost receivers by single-frequency relative kinematic positioning. Measurement Science and Technology 24(11), pp. 115010.

Sampietro, D., Caldera, S., Capponi, M. and Realini, E., 2017. Geoguard - an innovative technology based on low-cost gnss receivers to monitor surface deformations. 1st EAGE Workshop on Practical Reservoir Monitoring. 